\title{
Polarization of CMB and possible time dependence of dark energy
}

\author{
Noriaki Kitazawa \\ Department of Physics, Tokyo Metropolitan University, \\ Hachioji, Tokyo 192-0397, Japan \\ e-mail: noriaki.kitazawa@tmu.ac.jp
}

\begin{abstract}
Dark energy has been introduced to explain the present accelerating expansion of the universe. In the $\Lambda$ CDM model, the present standard model of cosmology, dark energy is described as a cosmological constant which is time independent. The possibility of the time dependence of dark energy has been investigated to obtain deeper understanding of it by looking redshift-magnitude relation of type Ia supernova, for example. We investigate the constraints to the time dependence of dark energy with several phenomenological models by fitting their parameters to the redshift-magnitude relation of the Pantheon supernova catalog, and confirm that little time dependence can not be excluded by the goodness-of-fit criterion only. Such a non-trivial time dependence causes different expansion rate in the period of reionization, which affects the low- $\ell$ polarization power spectrum of CMB. We investigate the possibility to detect the effect in future probe like LiteBIRD, for example. We find that the low- $\ell$ EE polarization power spectrum is enhanced in general, but it will be difficult to be detected as far as looking the EE polarization power spectrum only because of the limitation by cosmic variance.
\end{abstract}




\section{Introduction}

The nature of dark energy, which realizes the present accelerating expansion of the universe [1, 2], is unclear. Though it can be described as a cosmological constant in the $\Lambda$ CDM model, the present standard model of cosmology, it is simply a possible parameter and the origin is unknown. The cosmological constant can cause accelerating expansion of the universe with time-independent expansion rate. If dark energy is some kind of vacuum energy or some effect of modified gravity (for reviews, see [3, 4, 5], and also see [6] for a recent effort), the expansion rate can be time-dependent.

Since an absolutely convincing and well-motivated origin of dark energy has not been found yet, some phenomenological models of dark energy beyond the cosmological constant have been introduced, and they have been examined by cosmological observations. A typical phenomenological model is Chevallier-Polarski-Linder (CPL) model [7, 8] of non-trivial time dependence in the equation of state of dark energy $p=w \rho$ as

$$
w=w_{0}+w_{a}(1-a(t))
$$

where $w_{0}$ and $w_{a}$ are numerical constants, namely parameters, and $a(t)$ is the scale factor assuming flat Friedmann-Lemaitre-Robertson-Walker metric with the normalization of present value of the scale factor $a\left(t_{0}\right)=1$. The value of $w$ is $w_{0}$ at present, and it changes linearly to $w_{0}+w_{a}$ at very early time. The set of values of $w_{0}=-1$ and $w_{a}=0$ corresponds to the cosmological constant, or the $\Lambda$ CDM model. The model with $w_{a}=0$ keeping $w=w_{0}$ as a free parameter is called $w \mathrm{CDM}$ model. The typical constraints to these parameters are

$$
w_{0}=-0.939 \pm 0.073, \quad w_{a}=-0.31_{-0.24}^{+0.28}
$$

for CPL model, and

$$
w=-1.020 \pm 0.027
$$

for $w$ CDM model by using PLANCK CMB, Pantheon supernova, SDSS BAO+RSD and DES 
$3 \times 2$ pt data [9]. Though these results indicates that the $\Lambda$ CDM model is consistent with present data, the time dependence of $w$, namely $w_{a}$ has not been strongly constrained yet. It seems to be required to find some other way to constrain further the time dependence of $w$.

The non-trivial time dependence of dark energy changes the way of expansion of the universe not only in the era of dark energy dominance $(z<0.3)$, but also in the era of reionization $(6<$ $z<10$ ), though the dark energy contribution is not dominant in the latter era. Furthermore, the change of the values of cosmological parameters, like matter density $\Omega_{m}$, through the fit with data by observations in the region of $z<0.3$, where the effect of the non-trivial time dependence of dark energy is large, also changes the way of expansion in the era of reionization. This change affects the large-scale (low- $\ell$ ) power spectrum of the polarization of CMB, since the polarization is produced by the Thomson scattering with free electrons which are produced in the reionization process (see [10, 11, 12] for reviews). In this article we examine the possibility whether future precise measurements of the large-scale polarization power spectrum can give some constraints to the time dependence of dark energy.

We introduce the following two phenomenological models further, since the extrapolation of the CPL model to larger redshift region, beyond the era of dark energy dominance, is non-trivial and may be dangerous. The model of simple Taylor expansion

$$
w=w_{0}+w_{1} a(t)+\frac{1}{2} w_{2} a(t)^{2}
$$

with $w_{0}=-1$ naturally arrives at the case of the cosmological constant in early time, where $w_{1}$ and $w_{2}$ are free parameters. We call this model Taylor expansion model. We consider another simpler model with $w_{2}=0$ keeping $w_{1}$ as a free parameter, which we call linearCDM model. This model can be understood as a constrained CPL model.

In the next section we are going to obtain best-fit values of the parameters in these four models under the observed redshift-magnitude relation of type Ia supernova. We use the Pantheon catalog of type Ia supernova [13] 1]. In the analysis the binned data (40 redshift bins) are used. We use vary simple likelihood functions, since our aim is not to set the precise constraints to

\footnotetext{
${ }^{1}$ The data are open in public at http://dx.doi.org/10.17909/T95Q4X.
} 
these parameters, but to obtain typical reference values of parameters for the analyses of CMB polarization power spectrum. In section 3 we calculate low- $\ell$ EE polarization power spectrum in each phenomenological model and compare it to that in $\Lambda \mathrm{CDM}$ model. The power spectrum is numerically calculated by using CAMB [14] code. In the last section we conclude.

\section{Model parameters and redshift-magnitude relation}

The distance modulus of an object is defined by $\mu=m-M$, where $m$ is the apparent magnitude of the object and $M$ is the absolute magnitude assuming that the object were at distance $10 \mathrm{pc}$ from the observer. The relation between the luminosity distance $d_{L}$ to the object and the distance modulus is $\mu=5 \log \left(d_{L}[\mathrm{Mpc}]\right)+25$, where $d_{L}$ is measured in Mpc and the base of the logarithm is 10 . Consider the case that the light from the object is observed with cosmological redshift $z$. The luminosity distance to the object is given by

$$
d_{L}(z)=\frac{1+z}{H_{0}} \int_{0}^{z} d z^{\prime} \frac{1}{E\left(z^{\prime}\right)}
$$

where $H_{0}$ is the Hubble constant and $E(z) \equiv H(z) / H_{0}$ with $H(z)$ is the Hubble parameter as a function of redshift. The function $E(z)$ is described by model parameters:

$$
E(z)=\sqrt{\Omega_{m}(1+z)^{3}+\left(1-\Omega_{m}\right)(1+z)^{3\left(1+w_{0}+w_{a}\right)} e^{-3 w_{a} \frac{z}{1+z}}}
$$

for CPL and $w$ CDM models, and

$$
E(z)=\sqrt{\Omega_{m}(1+z)^{3}+\left(1-\Omega_{m}\right) e^{3 w_{1}\left(1-\frac{1}{1+z}\right)+\frac{3}{4} w_{2}\left(1-\frac{1}{(1+z)^{2}}\right)}}
$$

for Taylor expansion and linearCDM models. Here, we simply neglect the contribution of radiation, which is very small in the era of redshifts that we will investigate, and set the energy density of dark energy $\Omega_{\mathrm{DE}}=1-\Omega_{m}$ assuming flat universe. Then, the redshift-magnitude 

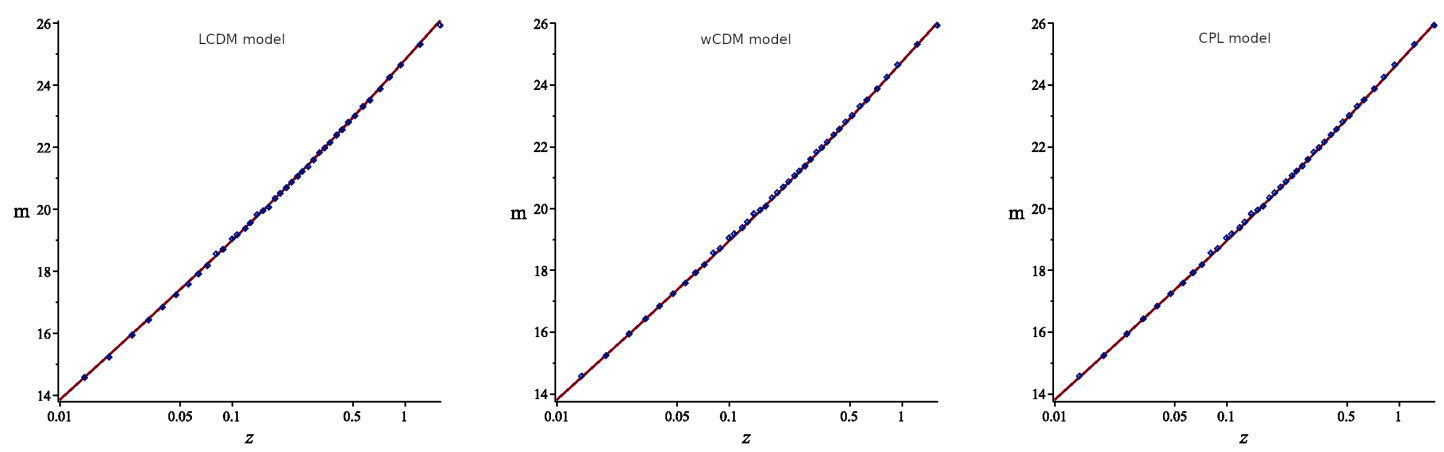

Figure 1: The Pantheon redshift-magnitude relation (40 redshift bins) fitted by three models: $\Lambda \mathrm{CDM}, w \mathrm{CDM}$ and CPL models from left to right. The Pantheon data are displayed as dots, which include error bars, and the lines are best fit results of corresponding three models. All the figures look the same and can not be distinguished with eyes.

Table 1: The best fit model parameters with Pantheon binned data. In addition to the best fit values of parameters in each model, minimum values of $\chi^{2}\left(\chi_{\min }^{2}\right)$ and corresponding reduced $\chi^{2}$ $\left(\chi_{\min }^{2} / \nu\right.$ with degrees of freedom $\left.\nu\right)$ are also given. The numbers in brackets mean fixed values.

\begin{tabular}{lccccccc}
\hline Model & $\Omega_{m}$ & $w_{0}$ & $w_{a}$ & $w_{1}$ & $w_{2}$ & $\chi_{\min }^{2}$ & $\chi_{\min }^{2} / \nu$ \\
\hline \hline$\Lambda$ CDM & 0.28 & $(-1)$ & - & - & - & 49.9 & 1.28 \\
$w$ CDM & 0.32 & -1.1 & - & - & - & 47.6 & 1.25 \\
$\mathrm{CPL}$ & 0.38 & -1.2 & -0.80 & - & - & 47.5 & 1.28 \\
linearCDM & 0.32 & $(-1)$ & - & -0.11 & - & 47.6 & 1.25 \\
Taylor & 0.35 & $(-1)$ & - & -0.7 & 1.1 & 47.3 & 1.28 \\
\hline
\end{tabular}

relation of type Ia supernova is described by

$$
m(z)=5 \log \left((1+z) \int_{0}^{z} d z^{\prime} \frac{1}{E\left(z^{\prime}\right)}\right)-5 a_{\mathrm{SN}}
$$

where $a_{\mathrm{SN}} \equiv \log \left(H_{0}\left[\mathrm{Mpc}^{-1}\right]\right)-5-M / 5$ is a constant for ideal type Ia supernova with a unique absolute magnitude. In fact the determination of Hubble constant from type Ia supernova data is achieved in principle by extracting $a_{\mathrm{SN}}$ from data by fitting redshift-magnitude relation with the knowledge of a unique absolute magnitudes of type Ia supernova (see [15] for a short review). In this article we do not determine the Hubble constant, but extracting model parameters as well as $a_{\mathrm{SN}}$ by fitting redshift-magnitude relation with eq.(8)).

The results of fitting the Pantheon binned data of redshift-magnitude relation by three models, $\Lambda$ CDM, $w$ CDM and CPL models, are shown in Fig. 1. The $\chi^{2}$ statistics for fitting is 
defined as

$$
\chi^{2} \equiv \sum_{i=1}^{40} \frac{\left(m_{i}-m\left(z_{i}\right)\right)^{2}}{\left(\sigma_{i}\right)^{2}},
$$

where $m_{i}, z_{i}$ and $\sigma_{i}$ are Pantheon data corresponding to the magnitudes, redshifts and errors of the magnitude, respectively. We adopt this simple one, since our aim is not to set precise constraints to model parameters, but to obtain typical reference values of model parameters for the analyses of CMB polarization power spectrum. Though each line in Fig. 1 is corresponding to the best fit values of model parameters, which make the value of $\chi^{2}$ minimum, it is impossible to distinguish each of them with eyes, since errors of the data are very small. The same happens for other two models, linearCDM and Taylor expansion models.

The summary of best fit values of parameters of each model is given in Table 1. The matter energy density parameter $\Omega_{m}$ is considered as a parameter in $\Lambda$ CDM model and also in other models. The ranges of scan of parameters are: $0.10 \leqq \Omega_{m} \leqq 0.50,-1.5 \leqq w_{0} \leqq-0.5$, $-1.0 \leqq w_{a} \leqq 1.0,-1.2 \leqq w_{1} \leqq 1.2,-1.2 \leqq w_{2} \leqq 1.2$ and $-5.0 \leqq a_{\mathrm{SN}} \leqq-3.0$. The best fit value of $a_{\mathrm{SN}}$ is equally -4.76 for all the models, that value correspond to $H_{0}=73.2[\mathrm{~km} / \mathrm{s} \mathrm{Mpc}]$ with $M=-19.263[\mathrm{mag}]$. The best fit values of $\Omega_{m}$ in the models with one parameter, $w \mathrm{CDM}$ and linearCDM models, are larger than that in $\Lambda$ CDM model. The same happens in the models with two parameters, CPL and Taylor expansion models, in which the best fit values of $\Omega_{m}$ are larger than those in one parameter models. In other words, better fitting the curve of redshiftmagnitude relation by many parameters results larger value of $\Omega_{m}$. However, as expected in Fig. 1, goodness-of-fit (reduced $\chi^{2}$ ) for each model is almost the same, and we can not choose the best model. It may indicate some limitation of this method to constrain model parameters, and we may need some new observables to go further. We are going to investigate the possibility of CMB polarization power spectrum in the next section.

\section{Polarization of CMB and time-dependent dark energy}

The time dependence of dark energy modifies the way of expansion of the universe in the period of reionization $(6<z<10)$, and it affects the CMB polarization at low- $\ell$ (large scales), 

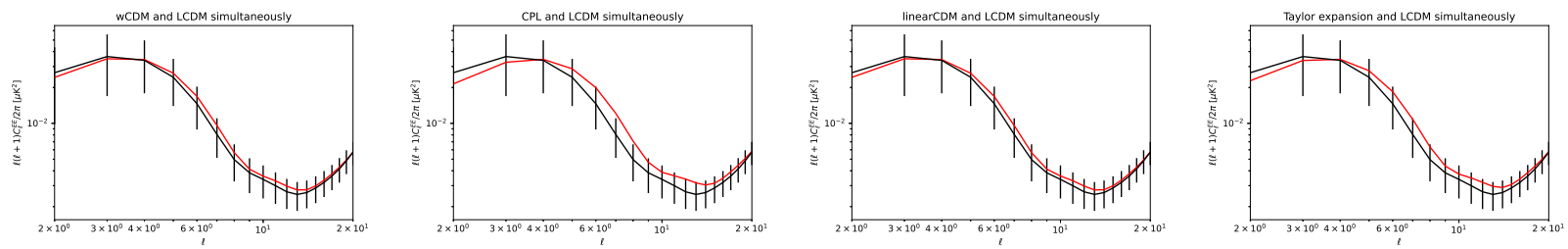

Figure 2: EE polarization power spectrum $D_{\ell}^{E E}=\ell(\ell+1) C_{\ell}^{E E} / 2 \pi$ with Pantheon binned data for $w \mathrm{CDM}$, CPL, linearCDM and Taylor expansion models, from left to right, respectively. Each figure includes two lines: predictions of $\Lambda$ CDM model (in black) and of phenomenological model (in red). The vertical lines in each figures show cosmic variance limited errors in the $\Lambda \mathrm{CDM}$ model. All the phenomenological models predict larger power for larger $\ell$.
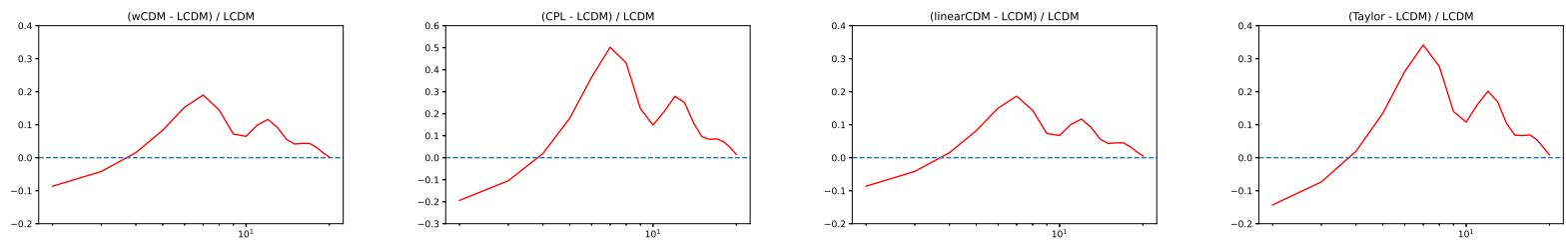

Figure 3: The differences of EE polarization power spectra between in phenomenological models and $\Lambda$ CDM model: $\left(D_{\ell}^{E E, \text { model }}-D_{\ell}^{E E, \Lambda \mathrm{CDM}}\right) / D_{\ell}^{E E, \Lambda \mathrm{CDM}}$ with the Pantheon binned data for $w \mathrm{CDM}$, CPL, linearCDM and Taylor expansion models, from left to right, respectively. There is a common qualitative pattern of $\ell$-dependent deviation from the prediction of $\Lambda \mathrm{CDM}$ model.

since the polarization is produced through the scattering with electrons which are produced in the process of reionization. Here, we concentrate on the EE polarization power spectrum at low- $\ell$, on which the cosmic-variance-limited measurement is expected in near future. Such a precise measurement may give further constraint or discovery of the time dependence of dark energy.

The EE polarization power spectrum $D_{\ell}^{E E}=\ell(\ell+1) C_{\ell}^{E E} / 2 \pi$ at low- $\ell$ for each model is numerically calculated using CAMB code with a set of basic cosmological parameters obtained by PLANCK [16]: $\Omega_{b} h^{2}=0.022, \tau=0.054, A_{s}=2 \times 10^{-9}$ and $n_{s}=0.965$. On $\Omega_{c} h^{2}$ we use the values of $\Omega_{m}$ by our fits with Hubble parameter $H_{0}=73.2[\mathrm{~km} / \mathrm{s} \mathrm{Mpc}]$, which is obtained from a supernova observation [17]. We can include a non-trivial equation of state of dark energy $w(a)$ in CAMB code using the option which sets $w(a)$ from numerical values.

The results with the best fit model parameters to the Pantheon binned data (Table 1) are shown in Figs. 2 and 3 . The black and red lines in each panel in Fig. 2 are EE polarization power spectra $(2<\ell<20)$ in the $\Lambda$ CDM model and phenomenological model, respectively. 

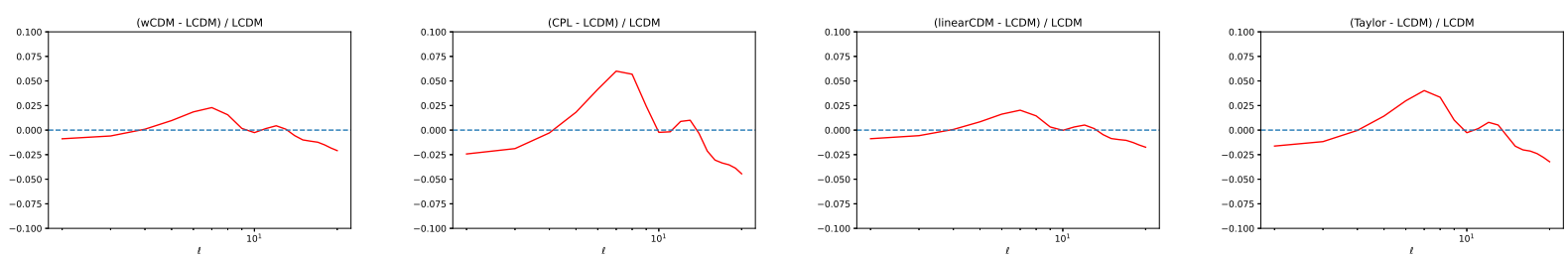

Figure 4: The same of Fig. 3, but in each figure the value of $\Omega_{m}$ in the $\Lambda$ CDM model is artificially set the value of each corresponding model.

The difference of EE polarization power spectrum in each model from that in the $\Lambda \mathrm{CDM}$ model is shown in Fig. 3. We see that the power are enhanced about order of $10 \%$ in all the phenomenological models in the range of $4<\ell<20$. This effect has been pointed out in [18] by semi-analytic calculations with some approximations. Fig. 3 indicates that the power spectra in $w \mathrm{CDM}$ and linearCDM model are almost the same. Note that the scale of vertical axes are different in the plots for CPL and Taylor expansion models in Fig. 3, though the shapes are almost the same. The largest enhancement of $50 \%$ happens in CPL model at $\ell=7$.

Since the difference of the EE polarization power spectrum of each model from that of the $\Lambda \mathrm{CDM}$ model is almost horizontal shift in $\ell$ direction, these results may be understood as the results of change in the angular diameter distance to reionization. The angular diameter distance is proportional to an integration of the inverse of eqs.(6) and (7), and the effect of each model is divided into two origins: the change of the value of $\Omega_{m}$ and the non-trivial redshift dependence of dark energy. Fig. 4 shows the differences of EE polarization power spectra between in each phenomenological model and $\Lambda \mathrm{CDM}$ model with the value of $\Omega_{m}$ in each corresponding model. We see that the magnitude of the difference is reduced by about a factor of ten and the original difference is in fact dominated by the change of the value of $\Omega_{m}$. The effect of the non-trivial redshift dependence of dark energy remains, even though it is small, which keeps the original shape, or $\ell$-dependence, of the difference. Note, however, that the origin of the different value of $\Omega_{m}$ in each model from that of the $\Lambda$ CDM model is the non-trivial redshift-dependence of dark energy in the fit with supernova redshift-magnitude relation.

Here, we show the necessity and importance of the precise knowledge of the reionization process. The typical present bound on the optical depth is $\tau=0.054 \pm 0.007$ [16]. The two 

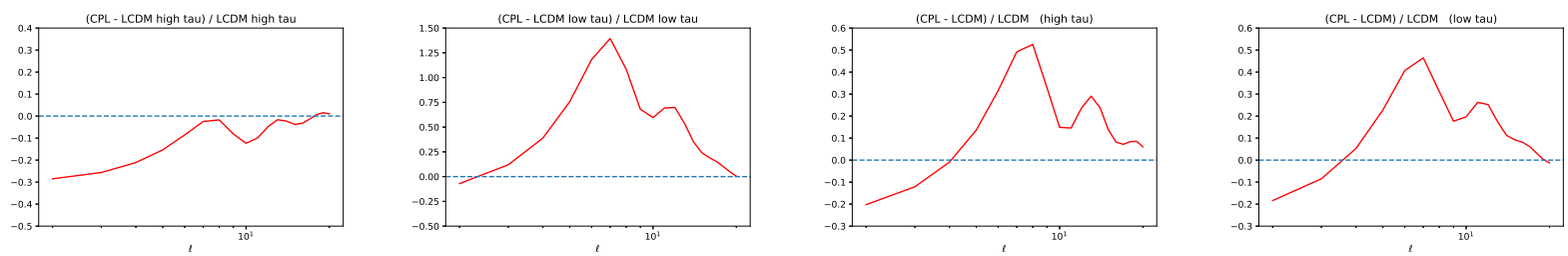

Figure 5: The differences of EE polarization power spectra between in CPL model and the $\Lambda \mathrm{CDM}$ model. In the left figure the value of the optical depth is set as $\tau=0.054+0.007$ in the $\Lambda \mathrm{CDM}$ model and in the middle-left figure it is set as $\tau=0.054-0.007$ in the $\Lambda$ CDM model keeping as $\tau=0.054$ in CPL model in both figures. In the middle-right and right figures, the value of the optical depth is set as $\tau=0.054+0.007$ and $\tau=0.054-0.007$, respectively, in both CPL model and the $\Lambda$ CDM model.

figures in Fig. 5 from the left show the differences of EE polarization power spectra in case of $\tau=0.054+0.007$ and $\tau=0.054-0.007$ in the $\Lambda \mathrm{CDM}$ model, respectively, keeping $\tau=0.054$ in CPL model. These figures show that the enhancement in the prediction of CPL model with $\tau=0.054$ is disappeared, if the $\Lambda$ CDM model with $\tau=0.054+0.007$ is correct in Nature, and the opposite happens, if the $\Lambda$ CDM model with $\tau=0.054-0.007$ is correct in Nature. It is clear that the precise understanding about the reionization process is certainly necessary. Fortunately, we can expect that the knowledge of reionization process will be extensively improved by observing 21-cm background by SKA [19] and other probes, for example, before the cosmic variance limited measurements of low- $\ell$ EE polarization power spectrum by LiteBIRD. Since the detailed quantitative evaluation of the uncertainty by ambiguous knowledge of reionization, like the work in [20, by forecasting the future ambiguity of the knowledge with various forthcoming observations is beyond the scope of this article, we leave it for future works. One naive possibility, which would be worth to be investigated in future, would be that the theoretical prediction of the difference of EE polarization power spectra between in some dark energy model and the $\Lambda$ CDM model would not be affected so much by the detail of the reionization process (see the right two figures in Fig. 5).

We introduce the following two quantities to investigate the detectability of this enhancement. One is the variance used in [21, 22, 23, 24]

$$
V_{\text {model }} \equiv \sum_{\ell=\ell_{\min }}^{\ell_{\max }}\left(\frac{2 \ell+1}{4 \pi}\right) C_{\ell}^{E E, \text { model }},
$$


Table 2: The quantities to measure the detectability of the enhancement in EE polarization power at low- $\ell$ in case of the Pantheon binned data. The degrees of freedom is defined as $\nu \equiv\left(\ell_{\max }-\ell_{\min }+1\right)-($ number of model parameter $)$.

\begin{tabular}{lccc}
\hline Model & $\Delta V_{\text {model }}$ & $\chi_{\text {model }}^{2}$ & $\chi_{\text {model }}^{2} / \nu$ \\
\hline \hline$w$ CDM & 0.447 & 1.28 & 0.0800 \\
$\mathrm{CPL}$ & 1.07 & 8.45 & 0.563 \\
linearCDM & 0.446 & 1.29 & 0.0807 \\
Taylor & 0.763 & 4.03 & 0.269 \\
\hline
\end{tabular}

which defines autocorrelation $C^{E E \text {,model }}(\theta=0)$ between $\ell_{\min }$ and $\ell_{\max }$. The error of this quantity is defined as

$$
\delta V^{2} \equiv \sum_{\ell=\ell_{\min }}^{\ell_{\max }}\left(\left(\frac{2 \ell+1}{4 \pi}\right) C_{\ell}^{E E, \text { model }} \sqrt{\frac{2}{2 \ell+1}}\right)^{2}
$$

where we assume that the error is cosmic-variance-limited. We define the significance of enhancement as

$$
\Delta V_{\text {model }} \equiv \frac{V_{\text {model }}-V_{\Lambda \mathrm{CDM}}}{\delta V}
$$

In this article we take $\ell_{\min }=4$ and $\ell_{\max }=20$. Another quantity is the measure of the differences in $D_{\ell}^{E E}$

$$
\chi_{\text {model }}^{2} \equiv \sum_{\ell=\ell_{\min }}^{\ell_{\max }} \frac{\left(D_{\ell}^{E E, \Lambda \mathrm{CDM}}-D_{\ell}^{E E, \text { model }}\right)^{2}}{\sigma_{\ell}^{2}},
$$

where

$$
\sigma_{\ell} \equiv D_{\ell}^{E E, \Lambda \mathrm{CDM}} \sqrt{\frac{2}{2 \ell+1}}
$$

is the error due to the cosmic variance only. This second quantity is simply the measure of the difference in $\ell$ by $\ell$ in comparison with error, or a sort of goodness-of-fit of the results in $\Lambda$ CDM model and phenomenological model.

The quantities $\Delta V_{\text {model }}, \chi_{\text {model }}^{2}$ and $\chi_{\text {model }}^{2} / \nu(\nu$ is the degrees of freedom) in case of the Pantheon binned data fits are summarized in Table 2. We see that the enhancement is small in comparison with error in each model. The differences of variances are less than $1 \sigma$, and fits between $D_{\ell}^{E E, \Lambda \mathrm{CDM}}$ and $D_{\ell}^{E E \text {,model }}$ are rather good. The effect in CPL model appears larger than those in linearCDM and Taylor expansion models. This would indicate that the extrapolation of the CPL model to larger redshift region could be problematic, because the expansion in 
$(1-a)=z /(1+z)$ may not be valid at large $z \sim 10$, though $a=1 /(1+z)$ remains small in Taylor expansion model. It will be difficult to measure this enhancement by the non-trivial time dependence of dark energy even in future precise measurements of CMB EE polarization power spectra by LiteBIRD [25], for example. Furthermore, since this phenomenon rather strongly depends on the knowledge of reionization process (the value of optical depth $\tau$, as we have seen, and reionization process (see [18] for quasi-quantitative arguments)), it will be required a global analysis relying on the consistency between many observables, including which will be available in future, not simply looking the low- $\ell$ EE polarization power spectrum only.

\section{Conclusions}

It has been shown that the redshift-magnitude relation of type Ia supernova still allows various non-trivial time-dependences of the equation of state of dark energy. The $\Lambda$ CDM model is not necessary the best model from the statistical point of view. Though the analysis in this article is rather simple one, some recent rigorous analysis give similar results: the constraints on the parameters is not strong enough in [9] and also [26] more recently, for example.

It would be worth to find other physical quantities which can give constraints to the time dependence of the equation of state of dark energy. We have investigated the possibility that the CMB polarization power spectrum at low- $\ell$ (large scales) can be such a quantity. This is because that the non-trivial time dependence of dark energy changes the way of expansion of the universe in the period of reionization, mainly through the change of the value of the matter density parameter $\Omega_{m}$ due to the fit with the redshift-magnitude relation of type Ia supernova, and the low- $\ell$ polarization is produced by the Thomson scattering with free electrons which are produced in the reionization process.

We have found that the CMB EE polarization power spectrum is enhanced around $4<\ell<$ 20 in comparison with the prediction of $\Lambda \mathrm{CDM}$ model in rather model independent way. However, the amount of enhancement is rather smaller in comparison with the ambiguity or error due to cosmic variance. Therefore, it will be difficult to constrain non-trivial time dependence of dark energy by simply looking the low- $\ell$ EE polarization power spectrum only. In case that 
the tensor-to-scalar ratio would be large and BB polarization power spectrum at low- $\ell$ would be observed in future, it would be an important observable to constrain the non-trivial time dependence of dark energy. The future efforts to understand the reionization process more precisely (see [27] in CMB, for example) are certainly very important. The future information on the cosmology at high redshift $(6<z<10)$ by $21-\mathrm{cm}$ and other observations will be very important also. Especially, the expansion history of the universe at high redshift is very important for clarifying the nature of dark energy (see [28] for future prospects, for example).

The same analysis using different supernova catalog, the Foundation Supernova Survey [29, 30], for example, would be very interesting to clarify whether the results of this article include some systematic error related with supernova data. A characteristic feature of the Foundation Supernova Survey is that they focus on the data of redshift region $z<0.1$ where the universe is dark energy dominant.

\section{Acknowledgments}

This work was supported in part by JSPS KAKENHI Grant Number 19K03851.

\section{References}

[1] A. G. Riess et al. [Supernova Search Team], "Observational evidence from supernovae for an accelerating universe and a cosmological constant," Astron. J. 116 (1998), 1009-1038 arXiv:astro-ph/9805201 [astro-ph]].

[2] S. Perlmutter et al. [Supernova Cosmology Project], "Measurements of $\Omega$ and $\Lambda$ from 42 high redshift supernovae," Astrophys. J. 517 (1999), 565-586 arXiv:astro-ph/9812133 [astro-ph]].

[3] A. Silvestri and M. Trodden, "Approaches to Understanding Cosmic Acceleration," Rept. Prog. Phys. 72 (2009), 096901 [arXiv:0904.0024 [astro-ph.CO]]. 
[4] T. Clifton, P. G. Ferreira, A. Padilla and C. Skordis, "Modified Gravity and Cosmology," Phys. Rept. 513 (2012), 1-189 [arXiv:1106.2476 [astro-ph.CO]].

[5] A. Joyce, B. Jain, J. Khoury and M. Trodden, "Beyond the Cosmological Standard Model," Phys. Rept. 568 (2015), 1-98 arXiv:1407.0059 [astro-ph.CO]].

[6] M. Raveri, "Reconstructing Gravity on Cosmological Scales," Phys. Rev. D 101 (2020) no.8, 083524 arXiv:1902.01366 [astro-ph.CO]].

[7] M. Chevallier and D. Polarski, "Accelerating universes with scaling dark matter," Int. J. Mod. Phys. D 10 (2001), 213-224 [arXiv:gr-qc/0009008 [gr-qc]].

[8] E. V. Linder, "Exploring the expansion history of the universe," Phys. Rev. Lett. 90 (2003), 091301 arXiv:astro-ph/0208512 [astro-ph]].

[9] S. Alam et al. [eBOSS], "Completed SDSS-IV extended Baryon Oscillation Spectroscopic Survey: Cosmological implications from two decades of spectroscopic surveys at the Apache Point Observatory," Phys. Rev. D 103 (2021) no.8, 083533 arXiv:2007.08991 [astroph.CO]].

[10] A. Kosowsky, "Cosmic microwave background polarization," Annals Phys. 246 (1996), 49-85 arXiv:astro-ph/9501045 [astro-ph]].

[11] W. Hu and M. J. White, "A CMB polarization primer," New Astron. 2 (1997), 323 arXiv:astro-ph/9706147 [astro-ph]].

[12] P. Cabella and M. Kamionkowski, "Theory of cosmic microwave background polarization," arXiv:astro-ph/0403392 [astro-ph]].

[13] D. M. Scolnic, D. O. Jones, A. Rest, Y. C. Pan, R. Chornock, R. J. Foley, M. E. Huber, R. Kessler, G. Narayan and A. G. Riess, et al. "The Complete Light-curve Sample of Spectroscopically Confirmed SNe Ia from Pan-STARRS1 and Cosmological Constraints from the Combined Pantheon Sample," Astrophys. J. 859 (2018) no.2, 101 arXiv:1710.00845 [astro-ph.CO]]. 
[14] A. Lewis, A. Challinor and A. Lasenby, "Efficient computation of CMB anisotropies in closed FRW models," Astrophys. J. 538 (2000), 473-476 arXiv:astro-ph/9911177 [astroph]].

[15] G. Efstathiou, "To H0 or not to H0?," arXiv:2103.08723 [astro-ph.CO]].

[16] N. Aghanim et al. [Planck], "Planck 2018 results. VI. Cosmological parameters," Astron. Astrophys. 641 (2020), A6 [arXiv:1807.06209 [astro-ph.CO]].

[17] A. G. Riess, S. Casertano, W. Yuan, J. B. Bowers, L. Macri, J. C. Zinn and D. Scolnic, "Cosmic Distances Calibrated to 1\% Precision with Gaia EDR3 Parallaxes and Hubble Space Telescope Photometry of 75 Milky Way Cepheids Confirm Tension with $\Lambda$ CDM," Astrophys. J. Lett. 908 (2021) no.1, L6 [arXiv:2012.08534 [astro-ph.CO]].

[18] N. Kitazawa, "Polarizations of CMB and the Hubble tension," arXiv:2010.12164 [astroph.CO]].

[19] D. J. Bacon et al. [SKA], "Cosmology with Phase 1 of the Square Kilometre Array: Red Book 2018: Technical specifications and performance forecasts," Publ. Astron. Soc. Austral. 37 (2020), e007 [arXiv:1811.02743 [astro-ph.CO]].

[20] M. Millea and F. Bouchet, "Cosmic Microwave Background Constraints in Light of Priors Over Reionization Histories," Astron. Astrophys. 617 (2018), A96 arXiv:1804.08476 [astro-ph.CO]].

[21] C. Monteserin, R. B. B. Barreiro, P. Vielva, E. Martinez-Gonzalez, M. P. Hobson and A. N. Lasenby, "A low CMB variance in the WMAP data," Mon. Not. Roy. Astron. Soc. 387 (2008), 209-219 [arXiv:0706.4289 [astro-ph]].

[22] M. Cruz, P. Vielva, E. Martinez-Gonzalez and R. B. Barreiro, "Anomalous variance in the WMAP data and Galactic Foreground residuals," Mon. Not. Roy. Astron. Soc. 412 (2011), 2383 [arXiv:1005.1264 [astro-ph.CO]]. 
[23] A. Gruppuso, P. Natoli, F. Paci, F. Finelli, D. Molinari, A. De Rosa and N. Mandolesi, "Low Variance at large scales of WMAP 9 year data," JCAP 07 (2013), 047 arXiv:1304.5493 [astro-ph.CO]].

[24] A. Gruppuso, N. Kitazawa, M. Lattanzi, N. Mandolesi, P. Natoli and A. Sagnotti, "The Evens and Odds of CMB Anomalies," Phys. Dark Univ. 20 (2018), 49-64 arXiv:1712.03288 [astro-ph.CO]].

[25] M. Hazumi et al. [LiteBIRD], "LiteBIRD: JAXA's new strategic L-class mission for allsky surveys of cosmic microwave background polarization," Proc. SPIE Int. Soc. Opt. Eng. 11443 (2020), 114432F [arXiv:2101.12449 [astro-ph.IM]].

[26] T. M. C. Abbott et al. [DES], "Dark Energy Survey Year 3 Results: Cosmological Constraints from Galaxy Clustering and Weak Lensing," arXiv:2105.13549 [astro-ph.CO]].

[27] D. J. Watts, G. E. Addison, C. L. Bennett and J. L. Weiland, "Beyond optical depth: Future determination of ionization history from the CMB," [arXiv:1910.00590 [astro-ph.CO]].

[28] N. Sailer, E. Castorina, S. Ferraro and M. White, "Cosmology at high redshift - a probe of fundamental physics," arXiv:2106.09713 [astro-ph.CO]].

[29] R. J. Foley, D. Scolnic, A. Rest, S. W. Jha, Y. C. Pan, A. G. Riess, P. Challis, K. C. Chambers, D. A. Coulter and K. G. Dettman, et al. "The Foundation Supernova Survey: Motivation, Design, Implementation, and First Data Release," Mon. Not. Roy. Astron. Soc. 475 (2018) no.1, 193-219 [arXiv:1711.02474 [astro-ph.HE]].

[30] D. O. Jones et al. [FSS], "The Foundation Supernova Survey: Measuring Cosmological Parameters with Supernovae from a Single Telescope," Astrophys. J. 881 (2019), 19 arXiv:1811.09286 [astro-ph.CO]]. 